\title{
Energiesystemtransformation - räumliche Politik und Stromnetzplanung
}

\author{
Jörg Fromme ${ }^{1}$
}

Eingegangen: 27. Juli 2015 / Angenommen: 17. Mai 2016 / Online publiziert: 7. Juni 2016

(c) Springer-Verlag Berlin Heidelberg 2016

Zusammenfassung Die Energiepolitik tendiert dazu, den Ausgleich zwischen der Erzeugung und dem Verbrauch von Energie möglichst großräumig zu organisieren und die Standortwahl für neue Kraftwerke und Speicher dem betriebswirtschaftlichen Kalkül im europaweiten Wettbewerb zu überlassen, wobei Netzkosten ausschließlich von den Stromverbrauchern gezahlt werden. Dies konzentriert die Erzeugung auf Gunststandorte und bedingt einen sehr weitgehenden Ausbau der Übertragungsnetze. In der Zivilgesellschaft werden mit der Energiewende dagegen oft Zielvorstellungen einer verbrauchsnahen Erzeugung und einem eher kleinräumig organisierten Ausgleich zwischen Erzeugung und Verbrauch verbunden. Von einer solchen „dezentralen Energiewende" versprechen sich die Befürworter einen verringerten Netzausbaubedarf.

Der Beitrag befasst sich mit den Hintergründen und der Praxis der Planungen zum Ausbau der Übertragungsnetze in Deutschland als einem Kristallisationspunkt für die räumliche Energiepolitik im Bereich der Stromversorgung. Eine besondere Rolle nimmt in dieser Hinsicht die Bedarfsplanung ein. Sie beruht unmittelbar auf energiepolitischen Zielen und Maßnahmen und stellt damit das Bindeglied zwischen Energiepolitik und Netzplanung dar. Die Bundesnetzagentur hat die Sensitivitätsanalyse als ein Instrument etabliert, das dazu dient, politische Handlungsoptionen im Hinblick auf ihre Wirkungen auf den Netzausbaubedarf im laufenden Bedarfsplanverfahren zu überprüfen. Dieses Instrument könnte auch genutzt werden, um auf der

Dr.-Ing. Jörg Fromme

joerg.fromme@tu-dortmund.de

1 Fakultät Raumplanung, Fachgebiet Ver- und Entsorgungssysteme, Technische Universität Dortmund, 44221 Dortmund, Deutschland
Basis fundierter Wirkungsanalysen unterschiedliche Transformationspfade systematisch in die netzplanerische und zugleich energiepolitische Abwägung einzustellen und so einen ganzheitlichen Steuerungsansatz mit integrierter Öffentlichkeitsbeteiligung zu verfolgen.

Schlüsselwörter Bedarfsplanung · Übertragungsnetzausbau · Energiewende · Regionalisierung $\cdot$ Zentralität

\section{Energy System Transition - Spatial Policy and Electricity Grid Planning}

Abstract Energy politics tend to organize the balance between power production and power consumption at the international scale as good as possible, leaving the site selection for new power or storage plants to pan-European competition. As grid costs are paid for by consumers, such policy causes an increasing spatial concentration of power plants on most beneficial sites and a very extensive transmission grid expansion. In civil society in contrast, the intent of "energy transition" is often linked with aims of placing power production units close to consumption to regional balance divergence between power production and consumption patterns. Supporters expect a minor transmission grid expansion requirement from such a "decentralized energy transition".

Against this background the article addresses the practice of grid planning in Germany as a focal point of the spatial energy policy. In this regard the role of transmission capacity planning is crucial. It is directly based on aims and measures of energy policy and thereby connects energy policy with transmission grid planning. The Federal Network Agency meanwhile has established sensitivity analysis as 
an instrument to check policy options with regard to their impacts on grid extension requirements. This instrument however could also be used to consider alternative paths in order to develop a systematic weighting regulation approach with integrated public participation based on wellgrounded impact analysis.

Keywords Capacity planning - Transmission grid expansion · Energy transition · Regionalisation · Centrality

\section{Einleitung}

Der geplante Ausbau der Strom-Übertragungsnetze wirkt gleichsam als Kristallisationspunkt für einen schwelenden Konflikt um die räumliche Ausgestaltung der Energiesystemtransformation. Maßgebliche Kräfte in Politik, Wirtschaft und Gesellschaft präferieren Konzepte einer zentralen Großversorgung mit ,(pan)-europäischer Integration“, wo „Strom überwiegend an Standorten erzeugt werden (soll), an denen die Ausbeute pro Flächeneinheit am größten ist" (vgl. Wachsmuth/Petschow/Brand et al. 2015: 9, 56, 61). Dabei werden Fluktuationen bei der Stromerzeugung aus Erneuerbaren Energien (EE) über die Vernetzung eines möglichst großen Gebiets ausgeglichen (vgl. Groscurth/Bode 2012: 7).

Dies kontrastiert mit dem Idealbild einer dezentralen Selbstversorgung ,in der Hand der Bürger“, bei der „Strom und Wärme überwiegend in Kleinanlagen in der Nähe der Verbraucher erzeugt und verbraucht werden“" (vgl. Wachsmuth/Petschow/Brand et al. 2015: 9, 56, 61). Technisch umgesetzt werden könnte ein eher kleinräumiger Ausgleich z. B. dadurch, dass ,dezentral möglichst viele kleine Zellen“ gebildet werden, ,die sich weitgehend autark versorgen, aber zur Absicherung mit den Nachbarzellen vernetzt sind“, und die ,die entsprechende Erzeugungskapazität und Speichermöglichkeiten, z. B. in Form von Bioenergie oder Batterien“ benötigen (Groscurth/Bode 2012: 7).

In der Wissenschaft, in der Bundespolitik und in der Energiewirtschaft besteht breiter Konsens darüber, dass die Energiewende nur erfolgreich umgesetzt werden kann, wenn sie als ,Ergebnis eines technisch-wirtschaftlichen Optimierungsprozesses“ (Plattform EE 2012: 6) gestaltet wird, der sich auf Ebene der Netznutzung durch einen möglichst uneingeschränkten Standort- und Technologiewettbewerb im Rahmen der europäischen Binnenmarktintegration vollzieht (vgl. z. B. Nicolosi 2012: 14 ff.). Dieser Wettbewerb setzt engpassfreie Netze voraus.

Aus der regionalen Energiewirtschaft heraus wird kritisiert, dass der geplante ,binnenmarktgetriebene Ausbau des Übertragungsnetzes“ über Jahrzehnte eine Struktur festschreibe, ,in der neue und innovative regionale Versorgungskonzepte deutlich weniger Chancen hätten“.
Marktanreize für ,eine sinnvolle Optimierung von lokalen und regionalen Versorgungsstrukturen“, die über die Nutzung von Speichern, Lastmanagement sowie Kraft-WärmeKopplung (KWK) einen Beitrag zur Versorgungssicherheit leisten könnten, wären nach umfassender Beseitigung der Netzengpässe durch Ausbau der Übertragungsnetze nicht mehr gegeben (vgl. N-ERGIE 2015: $1 \mathrm{f}$.).

Mautz/Byzio/Rosenbaum (vgl. 2008: 105, 148 ff.) deuten Entwicklungen, die mit der Markt- und Systemintegration der EE einhergehen, als eine ,Zentralisierung des Dezentralen“ und sehen die Energiewende an einem „Scheideweg“. Eine solche Wahrnehmung prägt auch die Proteste gegen den Übertragungsnetzausbau. So spricht sich der „Bundesverband der Bürgerinitiativen gegen den SüdLink“ unter dem Motto „JA zur Energiewende, NEIN zur Stromautobahn“ für ,eine dezentrale Energieplanung bzw. Energiepolitik als tragfähige Alternative" gegen eines der größten aktuellen Leitungsbauvorhaben aus (vgl. Bundesverband der Bürgerinitiativen gegen den SüdLink 2014).

Aber auch einige Energieökonomen äußern - wenn auch aus anderen Motiven - Zweifel am Sinn einer umfassenden Beseitigung von Netzengpässen durch Netzausbau. Schon im Rahmen einer Studie zum sogenannten ,Zielnetz 2050“ (vgl. Deutscher Bundestag 2011: 68) kommen die Gutachter zu dem Schluss, dass insbesondere bei einer europaweiten Betrachtung eine engpassfreie Netzauslegung zu ,wirtschaftlich nicht tragbarem Netzausbaubedarf führen“ könne (Fuchs/Mittelstaedt/Natemeyer et al. 2012: 60). Vor dem Hintergrund ähnlicher Überlegungen kritisiert die Monopolkommission, dass der Netzausbau in der öffentlichen Diskussion als alternativlos dargestellt werde. Sie weist darauf hin, dass ,der konkrete Umfang des Netzausbaus auf Basis einzelner Szenarien ganz entscheidend davon“ abhänge, ,ob und - wenn ja - in welchem Umfang Alternativen zum Netzausbau in die Planungen einbezogen werden“ würden (vgl. Monopolkommission 2013: 172 ff.).

Üblicherweise liegen der Netzplanung Prämissen zugrunde, die auf einem extern vorgegebenen fixen energiepolitischen Zielsystem und auf rechtlichen Status-quoBedingungen beruhen. Der Abschichtungseffekt, den die kaskadenartig gestuften Entscheidungsprozesse bewirken, erschwert es erheblich, diese den Bedarf maßgeblich prägenden Prämissen innerhalb der Verfahren auf den Prüfstand $\mathrm{zu}$ stellen oder gar zu beeinflussen. Grünwald (2015: 55) kommt vor diesem Hintergrund zu der pessimistischen Einschätzung, dass ,eine gesamtwirtschaftliche (und ökologische) Optimierung der eng miteinander verbundenen Systeme Erzeuger - Netz - Verbraucher nicht angesteuert werden" kann. Nach Auffassung der Monopolkommission fehle es im derzeitigen Regulierungssystem an einer ,sinnvollen Koordination zwischen Erzeugungsstandorten einerseits und Verbrauchszentren andererseits“ (vgl. Monopolkommission 2013: 172 ff.). 
In der Literatur werden verschiedene Vorschläge diskutiert, die darauf abzielen, den Zubau von Stromerzeugungskapazitäten räumlich-planerisch zu steuern und auf diese Weise besser mit dem Netzausbau zu koordinieren. Nach Vorstellungen der Akademie für Raumforschung und Landesplanung (ARL) soll der Bund den gesetzlichen Grundsatz in $\S 2$ Abs. 2 Nr. 6 Satz 8 ROG, wonach die Raumordnung die räumlichen Voraussetzungen für den EE-Ausbau $\mathrm{zu}$ schaffen hat, für das gesamte Bundesgebiet in einem Raumordnungsplan konkretisieren. Die Raumordnung soll dadurch zu einer verbesserten Abstimmung der Standortplanung für EE, deren Speicherung und der Netzausbauplanung beitragen. Dabei soll die Energiewende ,stärker als bisher Aspekte einer dezentralen Versorgung berücksichtigen“. Dies soll ,auch zur Minimierung der Belastungen der Bevölkerung und des Landschaftsbildes durch weitere Stromtrassen" beitragen (ARL 2014: 10 f.).

Während die ARL lediglich den EE-Ausbau raumordnerisch steuern und mit dem Netzausbau koordinieren will, nimmt Hermes (2014) darüber hinaus auch die Standortsteuerung für konventionelle Kraftwerke in den Blick. Weil nach seiner Auffassung die „tendenziell schwache Raumordnung des Bundes“" mit der Aufgabe einer räumlichen Gesamtkoordination der Energiewende überfordert wäre, schlägt er die Etablierung einer Energiefachplanung vor und rückt dabei das Bedarfsplanungsinstrumentarium in den Fokus. Dem Netzentwicklungsplan (vgl. § 12b Energiewirtschaftsgesetz (EnWG)) will er einen „Erzeugungsentwicklungsplan“ zur Seite stellen. Die Prämissen für die Erzeugungs- und Netzentwicklungsplanung sollen im Vorfeld ,unter Beteiligung der Übertragungsnetzbetreiber, potenzieller Erzeuger (Verbände), der Länder und der Öffentlichkeit unter vorbereitender Koordinierung der Bundesnetzagentur" erarbeitet und von der Bundesregierung beschlossen werden (Hermes 2014: vgl. 268 f.).

Alternativ dazu werden in der Energiewissenschaft Konzepte diskutiert, die statt einer unmittelbaren räumlichen Standortsteuerung eine Anpassung des Regulierungsrahmens vorsehen, um so Stromtransportkosten bei der Erzeugung einzupreisen und dadurch indirekt auch Standortentscheidungen $\mathrm{zu}$ beeinflussen (vgl. Monopolkommission 2013: $178 \mathrm{ff}$.).

Alle diskutierten Modelle lassen die Frage unbeantwortet, auf welche Weise die jeweiligen Entscheidungsträger Steuerungseingriffe im Hinblick auf deren Wirkung auf den Netzausbaubedarf bzw. auf das Gesamtoptimum eines zukünftigen Stromversorgungssystems konzipieren sollen. Noch Ende 2011 hatte die Bundesnetzagentur darauf hingewiesen, dass es ,gegenwärtig fachlich nicht geklärt“ sei, „wie sich einzelne Parameterausprägungen“ bei den Planungsprämissen „kausal auf den Netzentwicklungsbedarf auswirken“. In diesem Sinne könne im Vorgriff auf Netzberechnungen, wie sie die Übertragungsnetzbetreiber im Zuge der Netzentwicklungsplanung durchführen, keine Aussage darüber getroffen werden, welche konkreten Annahmen ,zu einem optimierten, das heißt minimalen, Netzausbau führen werden“ (BNetzA 2012a: 32). Demnach können entsprechende Weichenstellungen z. B. zur Standortverteilung von Kraftwerkskapazitäten allenfalls in einem iterativen politischen Willensbildungs- und Planungsprozess gefunden werden, der Wirkungsanalysen einschließt.

In diesem Beitrag wird das bestehende Bedarfsplanungsinstrumentarium - dem Vorschlag von Hermes insoweit folgend - als Anknüpfungspunkt für die Entwicklung und Implementierung eines ganzheitlich optimierenden Steuerungsansatzes betrachtet. Dabei liegt der Schwerpunkt auf der Frage danach, ob und wie verfahrensextern gesetzte energiepolitische Rahmenbedingungen, die den Netzausbaubedarf maßgeblich prägen, innerhalb der Planungsverfahren auf den Prüfstand gestellt werden bzw. gestellt werden können.

Dieser Beitrag befasst sich zunächst (Kapitel 2) mit aktuellen energiepolitischen Rahmenbedingungen im Spannungsfeld zwischen Energiewende, Binnenmarktintegration und EE-Ausbauförderung, die als Prämissen auch Eingang in die Netzplanung finden. Zudem werden Konzepte zur gezielten Allokation der Netznutzungen und des Netzausbaubedarfs sowie Konzepte zum regionalen Ausgleich zwischen Stromnetzeinspeisungen und -entnahmen als Optionen zur Minderung des Netzausbaubedarfs diskutiert.

Kapitel 3 stellt die Bedarfsermittlung für den Netzausbau im Spannungsfeld zwischen Marktprognose und energiepolitischer Steuerung dar. Zur Einführung wird ein kurzer Überblick über das Instrumentarium der Bedarfsplanung gegeben. Im Anschluss daran wird die aktuelle Planungspraxis analysiert. Betrachtet werden dabei zunächst die Erstellung von Netznutzungsszenarien sowie die netzplanerische Abwägung. Schließlich wird die Integration der Sensitivitätsanalyse in den Bedarfsplanungsprozess als ein Ansatz zur flexiblen wechselseitigen Anpassung zwischen Bedarfsplanung und energiepolitischer Rahmensetzung vorgestellt. Kapitel 4 skizziert Schlussfolgerungen zur Unterstützung einer ganzheitlichen Steuerung des Stromversorgungssystems auf Bedarfsplanungsebene.

Der Beitrag stellt die persönliche Sichtweise des Autors dar. Er beruht auf einer Auswertung von Fachpublikationen und von Stellungnahmen zu Netzplanungs-Konsultationsverfahren, die im Rahmen des vom Bundeswirtschaftsministerium geförderten Forschungsvorhabens „Interdisziplinäres Forschungsprojekt Stromnetzplanung " (Förderkennzeichen 03ET7501 A) durchgeführt wurde. Weitere wichtige Impulse kamen aus der Mitarbeit an dem Modellvorhaben der Raumordnung (MORO) „Regionale Energiekonzepte als strategisches Instrument der Landes- und Regionalplanung" des Bundesinstituts für Bau-, Stadt- und Raumforschung (BBSR) (vgl. BMVI 2015). 


\section{Die räumliche Entwicklung des Stromversorgungssystems im Spannungsfeld zwischen Energiewende, Liberalisierung und Binnenmarktintegration}

In diesem Kapitel werden zunächst (2.1) verschiedene Merkmale der Zentralität der Stromversorgung in der Energiewende dargestellt. Auf dieser Grundlage wird dann beschrieben, wie die Liberalisierung und Integration der europäischen Energiemärkte die zukünftige räumliche Entwicklung des Stromversorgungssystems unter diesem Aspekt prägen (2.2). Im Anschluss daran (2.3) werden Rahmenbedingungen für die Allokation der EE-Stromerzeugung vor dem Hintergrund einer zunehmenden europäischen Harmonisierung der Ausbau-Förderung dargelegt. Schließlich wird der Stand der Forschung zum Zusammenhang zwischen der Allokation der Netznutzungen und dem Netzausbaubedarf skizziert (2.4). Kapitel 2 zeigt damit zugleich die Prämissen der Netzplanung ebenso wie den Diskursrahmen für die Reduzierung des Netzausbaubedarfs auf.

\subsection{Merkmale der Zentralität der Stromversorgung in der Energiewende}

In einem Stromversorgungssystem, das im Wesentlichen auf der Nutzung fluktuierender erneuerbaren Energien (fEE) in Form von Wind- und solarer Strahlungsenergie basieren soll, müssen die übrigen Systemkomponenten an die zeitlich unflexible Stromlieferung aus fEE angepasst werden, um jederzeit die Residuallast decken zu können. Als Residuallast bezeichnet man die Verbrauchslast abzüglich der fEE-Netzeinspeiseleistungen. Zu den Flexibilisierungsoptionen zählen - abgesehen vom Netzausbau - die Stromspeicherung, das Last- und Erzeugungsmanagement und die Anpassung der Reaktionsfähigkeit und -geschwindigkeit des steuerbaren Backup-Kraftwerksparks (vgl. BMVI 2015: 179 ff.). Entscheidende anlagenbezogene technische Zentralitätsparameter sind nach Bauknecht/Vogel/Funcke (vgl. 2015: 7 ff.) die Anschlussnetzebenen und die Standortverteilungen der zukünftigen Versorgungsinfrastruktur sowohl bezogen auf Anlagen zur Stromerzeugung als auch auf die zur Flexibilisierung. Als weiterer wichtiger Zentralitätsparameter gilt die räumliche Ebene, auf der das Stromversorgungssystem gesteuert wird.

Bauknecht/Vogel/Funcke (vgl. 2015: 9) stufen technische Anlagen unter dem Gesichtspunkt der Kapazitätsgrößen und der Netzanschlussebenen dann als zentral ein, wenn sie unmittelbar an das Übertragungsnetz angeschlossen sind. $\mathrm{Zu}$ den zentralen Erzeugungsanlagen gehören demnach auch Offshore-Windparks oder große Wasserbzw. solarthermische Kraftwerke. Solche Anlagen werden zudem auch deshalb als zentral betrachtet, weil sie gebün- delt an Gunststandorten errichtet werden und sich daher dort hohe Kapazitäten räumlich konzentrieren.

Dagegen sind dezentrale Anlagen dadurch gekennzeichnet, dass sie aufgrund kleinerer Anschlussleistungen an Verteilnetze entweder auf Niederspannungs-, Mittel- oder Hochspannungsebene angebunden werden. Zudem sind Versorgungseinrichtungen als desto dezentraler einzustufen, je mehr sie in Bezug auf Kapazitätsgröße und Betriebsweise an den Bedarf im räumlichen Umfeld angepasst sind. Als Extremform dezentraler Konzepte können in diesem Sinne autarke Eigenversorgungen gelten, die z. B. auf Fotovoltaik-Batterie-Systemen oder auf Kraft-WärmeKopplungsanlagen mit angeschlossenem Wärmespeicher beruhen.

Die Frage, ob Betreiber sich bei der Fahrweise ihrer Anlagen an der jeweiligen Verbrauchssituation in ihrem unmittelbaren Umfeld orientieren, hängt wesentlich von der räumlichen Ebene ab, auf der der erforderliche Ausgleich zwischen Erzeugung und Verbrauch organisiert wird bzw. sogenannte Systemdienstleistungen wie die Frequenz- und Spannungshaltung erbracht werden. Ein großräumiger Ausgleich ist ein Charakteristikum eines zentralisierten Stromversorgungssystems, während ein eher regionaler Ausgleich als Kennzeichen eines dezentralen Systems gelten kann (vgl. Bauknecht/Vogel/Funcke 2015: 10).

\subsection{Liberalisierung und europäische Binnenmarktintegration}

Die Liberalisierung führte seit ihrem Beginn Mitte der 1990er-Jahre allmählich zu einem Verlust der räumlichen Bindungen in der Elektrizitätswirtschaft. Für ehemals vertikal integrierte und durch Gebietsmonopole geschützte Versorgungsunternehmen greifen Entflechtungsvorschriften bezogen auf die Wertschöpfungsstufen Stromerzeugung, Stromhandel, Stromtransport, Stromverteilung und Vertrieb. Nur noch die Netzwirtschaft gilt als natürlicher Monopolbereich und ist staatlich reguliert, während die übrigen Wertschöpfungsstufen dem Wettbewerb unterliegen.

Im Zuge der Liberalisierung und Binnenmarktintegration werden zudem die Marktgebiete sukzessive vergrößert, sodass auch der räumliche Zusammenhang zwischen Erzeugung, Versorgung und Verbrauch sich immer mehr auflösen wird. In liberalisierten Märkten können Kraftwerke oder Speicher grundsätzlich vollkommen unabhängig von der räumlichen Entfernung zu Verbrauchern oder von lokalen Netzengpässen gebaut und betrieben werden. Dies gewährleistet ein transaktionsunabhängiges Netzentgeltsystem (vgl. König 2013: 74 ff., 92) mit entfernungsunabhängiger Gestaltung (,Briefmarkentarif“) und ausschließlicher Trägerschaft der Netzkosten durch die Stromverbraucher (vgl. Hobohm/Koepp/Michelsen et al. 2006: 5). Marktsignale steuern den Anlagenbetrieb über zentrale Strombör- 
sen. Die wettbewerbliche Strompreisbildung setzt fiktional ein vollkommen engpassfreies Netz innerhalb sogenannter „Gebotszonen“ voraus und antizipiert damit eine vollständige Engpassbeseitigung durch Netzausbau nach dem Paradigma der (durchgehend engpassfreien) „Kupferplatte“ (vgl. Litz/Rosenkranz 2015: 11). Dies führt dort zu einem flächendeckend einheitlichen Großhandelspreis. An welchem Ort welche Anlage zu welchem Zeitpunkt welche Strommengen ins Netz einspeist oder speichert, richtet sich unter diesen Bedingungen nicht mehr nach regionalen Verbrauchsanforderungen, sondern ausschließlich nach der aktuellen Wettbewerbssituation innerhalb eines Marktgebiets.

Die 2010 begonnene und seitdem stufenweise fortschreitende Kopplung nationaler Strommärkte als ein Zwischenschritt zu einem europäischen Binnenmarkt (vgl. Litz/Rosenkranz 2015: 8) ermöglicht darüber hinaus eine einheitliche Marktpreisbildung über mehrere Gebotszonen hinweg sowie eine koordinierte Bewirtschaftung der Netzengpässe an den Grenzkuppelstellen zwischen den Mitgliedstaaten. Seit Anfang 2014 ist Deutschland in der sogenannten ,North-Western-Region“ (NWE) mit 18 weiteren nationalen Marktgebieten zusammengeschlossen. Diese Marktkopplung führt dazu, dass nun auch nicht mehr die jeweilige nationale Residuallast die nationale Erzeugung bestimmt, sondern die Lastsituation im transnationalen Marktgebietszusammenschluss. Dies trägt zu einer weiteren Zentralisierung des Stromversorgungssystems bei.

Der großräumige Netzverbund und die Marktintegration ermöglichen es, sowohl Verbrauchslastspitzen als auch Leistungsspitzen der fEE-Stromeinspeisung, die bei weiträumiger Standortverteilung der vernetzten Anlagenkapazitäten und Siedlungsräume ungleichzeitig auftreten, durch Überlagerung zu glätten (vgl. BMWi 2014: 33). Durch die auf diese Weise genutzten Ausgleichseffekte können bei gleicher fEE-Stromerzeugungsmenge und bei gleichem Niveau an Versorgungssicherheit Kraftwerks- und Speicherkapazitäten eingespart werden, die verfügbar sein müssen, wenn Windenergie- und Solaranlagen zeitweilig entweder Überschussstrom erzeugen oder nicht genug Strom liefern, um den momentanen Bedarf decken zu können (r2b/ISI/ CONNECT et al. 2014: 12).

Die bisherige energierechtlich fixierte, im nationalen Rahmen organisierte Absicherung gegen Versorgungsengpässe erfordert es, orientiert an nationalen Leistungsbilanzen inländische Kraftwerkskapazitäten aufzubauen. Um volkswirtschaftliche Effizienzpotenziale besser nutzen zu können, soll die Versorgungssicherheit zukünftig aber nicht mehr national, sondern zunehmend im europäischen Verbund gewährleistet werden (BMWi 2014: 34 f.). In gekoppelten Märkten werden dann nationale Kapazitätsreserven in dem gegebenen Umfang nicht mehr benötigt. Die im Zuge der Marktkopplung entstehenden Überkapazitäten werden durch Marktbereinigung nach und nach abgebaut.

\subsection{Binnenmarktintegration und Allokation des EE- Ausbaus}

Der internationale Standort- und Technologiewettbewerb soll perspektivisch auch EE-Kapazitäten betreffen. Dahinter steht die Leitvorstellung einer großräumigen Arbeitsteilung, bei der schwerpunktmäßig Solarenergie in Südeuropa und Nordafrika sowie Wasserkraft und Windenergie in Nordeuropa bzw. auf dem Meer erschlossen werden (vgl. ENTSO-E 2010: 160).

Mitgliedstaatliche EE-Ausbauziele und darauf abgestimmte nationale Anreizsysteme sind gemäß Richtlinie 2009/28/EG zwar europarechtlich legitimiert, geraten aber zunehmend unter den Einfluss von Harmonisierungsbestrebungen, wie sie aktuell in den neuen EU-Beihilfeleitlinien (vgl. EU-Kommission 2014) ihren Ausdruck finden (vgl. Müller 2014).

Die Bundesregierung hat sich bei der jüngsten Novelle des Erneuerbaren-Energien-Gesetzes (EEG), die im August 2014 in Kraft getreten ist, bereits teilweise an die Beihilfeleitlinien angepasst. Das Förderregime beruht aktuell im Wesentlichen noch auf gesetzlich fixierten Vergütungssätzen. Für 2017 ist die weitgehende Umstellung der Förderung auf ein Ausschreibungsmodell vorgesehen, wobei die Vergütungshöhe im Wettbewerb ermittelt werden soll. Im Vorfeld hatte es bereits eine entsprechende Umstellung für Freiflächenfotovoltaikanlagen gegeben, die eingeführt wurde, um so Praxiserfahrungen mit dem neuen Fördermodell sammeln zu können. Die Bundesregierung plant, im Rahmen der Ausschreibungen in enger Abstimmung mit interessierten Partnerländern auch ausländischen Strom zu fördern (vgl. BMWi 2015: 7).

Mit der Orientierung auf einen kontinentalen bzw. transkontinentalen Ausgleich durch großräumige Vernetzung geraten politische Konzepte unter Druck, die innerhalb Deutschlands eine verbrauchsnahe Stromerzeugung anstreben. So vertritt der Bundesverband der Energie- und Wasserwirtschaft e. V. (BdEW) die Auffassung, ,dass eine gegebenenfalls stärkere Förderung von Erneuerbaren Energien in Regionen Deutschlands, in denen einzelne Erzeugungstechnologien bislang einen geringen Ausbaugrad aufweisen (z.B. Windkraft in Süddeutschland) vor dem Hintergrund einer europäischen Harmonisierung der Fördermechanismen mit dem damit induzierten Wettbewerb um Standorte mit günstigen meteorologischen Bedingungen im Widerspruch“ stünden (BdEW 2012: 3). 


\subsection{Allokation der Netznutzung und Netzausbaubedarf}

Der Stromtransportbedarf hängt von der durchschnittlichen räumlichen Distanz zwischen den Standorten der Stromeinspeisung und der Stromentnahme ab (vgl. ÜNB 2012: 42 f.). Dies lässt Planungs- und Steuerungsansätze attraktiv erscheinen, die darauf hinauslaufen, die Netznutzung und den Netzausbau koordiniert zu steuern. Dies ist vor allem dann sinnvoll, wenn eine solche Koordination zu einer gesamtwirtschaftlichen Kostenreduktion führt, wie einige Energiewissenschaftler erwarten (vgl. Monopolkommission 2013: 111; Adam/Agsten/Benedix et al. 2013: 12; Bucksteeg/Niesen/Himmes et al. 2014: 166; Haucap/Pagel 2014: 6). Dazu müssten Einsparungen beim Netzbetrieb oder beim Netzausbau Mehrkosten überkompensieren, die bei der gezielten verbrauchsnahen Ansiedlung der Stromerzeugung (etwa für aufwendigeren Brennstofftransport bei Wärmekraftwerken) oder für Investitionen in höhere Anlagenkapazitäten (zum Ausgleich geringerer standortspezifischer Erträge bei der EE-Stromerzeugung) entstehen würden (vgl. Schuster 2013: 51 ff.). Ein netzdienliches Verhalten von Kraftwerks- und Speicherbetreibern setzt allerdings geeignete Anreizmechanismen voraus. Dazu gehören z. B. Netznutzungsentgelte für Kraftwerksbetreiber oder eine regionale Differenzierung der EE-Förderung (vgl. Haucap/ Pagel 2014).

Zum Januar 2016 hat der von der bayerischen Landesregierung politisch kanalisierte Protest die Bundesregierung veranlasst, einen Erdkabelvorrang für die großen Bauvorhaben zur Hochspannungsgleichstromübertragung (HGÜ) gesetzlich zu implementieren (vgl. § 3 Bundesbedarfsplangesetz (BBPIG)). Der Erdkabelvorrang soll die gesellschaftliche Akzeptanz in Bezug auf den Netzausbau verbessern und dadurch zu einer Beschleunigung der Verfahren beitragen. Er wird aber wohl den Übertragungsnetzausbau aller Voraussicht nach erheblich verteuern. Dies könnte dazu beitragen, bestimmten Netzausbaualternativen - wie z. B. einer stärker dezentral ausgerichteten Energieversorgung aus gesamtwirtschaftlicher Sicht Effizienzvorteile zu verschaffen gegenüber Transformationspfaden, die ein weitgehend engpassfrei ausgebautes Übertragungsnetz voraussetzen (vgl. CONSENTEC/Frontier 2008: 44).

\subsubsection{Standortoptimierung für konventionelle Kraftwerke}

Die Effizienz einer verbrauchsnahen Allokation konventioneller Kraftwerke wird von der Relation zwischen Brennstoff- und Stromtransportkosten beeinflusst. Braunkohlekraftwerke können aufgrund des geringen Heizwertes der Braunkohle wirtschaftlich nur an ausgesprochen reviernahen Standorten betrieben werden. Dagegen bietet die verbrauchsnahe Ansiedlung von Erdgaskraftwerken Potenziale zur Reduzierung der volkswirtschaftlichen Gesamt- kosten der Stromversorgung (vgl. CONSENTEC/Frontier 2008: 43; Schuster 2013: 97 ff.). Zum Teil gilt dies aber auch für Steinkohlekraftwerke im Vergleich verschiedener Binnenlandstandorte untereinander (vgl. CONSENTEC/ Frontier 2008: 43 f.).

\subsubsection{Standortoptimierung für die Wind- und Solarstromerzeugung}

Die Wirkungen einer verbrauchsnahen Wind- und Solarstromerzeugung auf den Netzausbaubedarf werden für sich genommen allgemein als eher gering eingeschätzt. Bucksteeg/Niesen/Himmes et al. (vgl. 2014: 189) gehen davon aus, dass verbrauchsnah installierte fEE-Erzeugungsanlagen Netzausbau in der Regel nicht vermeiden können, weil sie „nicht zwangsläufig zu den netzgünstigsten Zeiten einspeisen“.

Eine Studie im Auftrag der „AGORA Energiewende“ (einer gemeinsame Initiative der Stiftung Mercator und der European Climate Foundation) kommt zu dem Ergebnis, dass ein verbrauchsnaher Ausbau der Onshore-Windenergie- und Fotovoltaik-Kapazitäten im Vergleich zu einer Ansiedlung an ertragsbesten Standorten zu einer teilweisen Verlagerung des Netzausbaubedarfs in die Verbrauchszentren führen würde, ohne aber den Gesamtausbaubedarf deutlich zu verringern. In der gesamtwirtschaftlichen Bewertung führt die Zubau-Allokation an ertragsbesten Standorten zu Kostenvorteilen gegenüber der verbrauchsnahen Ansiedlung, weil die Kosteneinsparungen bei Netzausbau und -betrieb durch Mehrkosten, die bei - in der Summe gleich großer Stromerzeugung für die Errichtung von fEEAnlagen an ertragsschwächeren Standorten aufgewendet werden müssten, überkompensiert werden (vgl. Fürstenwerth/Bock/Tersteegen et al. 2013: 56 ff.). Die Mehrkosten für einen gegebenenfalls zukünftig erhöhten Erdkabelanteil im Übertragungsnetz sind in die Berechnungen allerdings nicht eingegangen.

Die Bundesnetzagentur gelangt, gestützt auf die AGORA-Studie, zu der Auffassung, dass eine verbrauchsnahe Erzeugung auf Basis Erneuerbarer Energien erst dann „einen nennenswerten Effekt auf den Netzausbaubedarf" habe, ,wenn auch konventionelle Kraftwerke verbrauchsnah verortet sind“" (vgl. BNetzA 2013: 41).

\subsubsection{Konzepte zum regionalen Ausgleich}

Statt den Ausgleich über einen möglichst großen Bilanzierungsraum zu realisieren, kann versucht werden, in „Energiezellen“ Erzeugung und Verbrauch so weit wie möglich auszubalancieren (vgl. Groscurth/Bode 2012: 7). Der ,zellulare Ansatz“ verspricht nicht nur einen höheren Selbstversorgungsgrad, mehr bürgerschaftliche Teilhabe und gesellschaftliche Akzeptanz, sondern auch eine stärke- 
re Konvergenz der unterschiedlichen Energieträger Strom, Gas und Wärme. Die Fähigkeit der Zellen zur automatisierten Steuerung, die auf Konzepten zur Selbstorganisation beruht, trägt darüber hinaus dazu bei, die Komplexität der Netzführung zu reduzieren. Sie gilt ansonsten zukünftig als nicht mehr beherrschbar aufgrund der weiteren Schwerpunktverlagerung der Stromerzeugung auf die unteren Netzebenen (Energieerzeugung aus EE und Einspeisung in Verteilnetze) und aufgrund der damit verbundenen $\mathrm{Zu}$ nahme bidirektionaler Leistungsflüsse über Netzebenen hinweg (durch Rückspeiseleistungen von durch EE-Anlagen eingespeister Leistung, die die lokale Stromnachfrage übersteigt) (vgl. VDE/ITG 2010: 56).

Damit die Erzeugung mit dem Verbrauch von Energie regional weitgehend ausgeglichen werden kann, müssten in den Zellen bedarfsangepasste Erzeugungskapazitäten aufgebaut werden. fEE-Überschüsse müssten gespeichert oder vor Ort für Wärme- und Mobilitätszwecke genutzt und Angebotslücken durch steuerbare Kraftwerke gedeckt werden. Die Bereitstellung entsprechender Kapazitäten wäre aber auch von der örtlichen Flächenverfügbarkeit abhängig. $\mathrm{Ob}$ genügend geeignete Flächen verbrauchsnah zur Verfügung stehen, ist für den EE-Ausbau eine Frage der ortsspezifischen Erträge, der Verbrauchslastdichte und der Gebietsgröße. Zumindest für Ballungsgebiete oder Industriestandorte wäre eine vollständig autarke Versorgung aufgrund fehlender Potenzialflächen vor Ort nicht darstellbar (vgl. Peter 2013: I).

Birkner (2014) plädiert daher dafür, gestaffelte teilautonome Energie-Cluster auf der Ebene einzelner Gebäude, Quartiere, Städte und Regionen zu schaffen. Sie seien ,als schalenförmige [...] integrierte Struktur zu konzipieren " und könnten nach dem „Prinzip der technischen Subsidiarität“ jeweils einen eigenständigen Beitrag zur Beherrschung der Volatilität leisten. Dazu wären „verschiedene Kraftwerke, also Windparks sowie Biomasse- oder Solaranlagen, über ein großes Gebiet möglichst homogen zu verteilen, auch wenn dadurch nicht die maximale Energieausbeute erzielt“" werde (Birkner 2014: 2).

Weil es aufgrund der regional ungleich verfügbaren EEPotenziale unter- und überbilanzierte und nur im Idealfall ausbilanzierte Energiezellen gäbe, wäre eine Energieübertragung zum Ausgleich zwischen mehreren Energiezellen auf jeden Fall erforderlich. Der Übertragungsbedarf könnte aber, so das Ergebnis einer VDE-Modellrechnung für eine 100-Prozent-EE-Vollversorgung für Deutschland, um 45 Prozent gegenüber einer vorrangigen Nutzung ertragsbester Standorte reduziert werden, wenn in einem so strukturierten Versorgungssystem die verbrauchsnahen EE-Potenziale gezielt genutzt werden würden (vgl. Benz/Dickert/ Erbert et al. 2015: $57 \mathrm{ff}$.).

Voraussetzungen für die Umsetzung des ,zellularen Ansatzes“ sind offenbar noch „Felderprobungen zur Machbar- keit“". Bislang ist außerdem noch ungeklärt, wer bei einer breiten Einführung unter welchen rechtlichen Voraussetzungen die „Verantwortung für Planung und Betrieb des Gesamtsystems“ übernehmen könnte (VDE 2015: 2).

\section{Die Netzausbaubedarfsermittlung - zwischen Marktprognose und politischer Steuerung}

Vor dem skizzierten Hintergrund aktueller politischer Maßnahmen und Trends zur räumlichen Gestaltung der Energiesystemtransformation soll nun das Instrumentarium der Netzausbaubedarfsermittlung im Spannungsfeld zwischen Marktprognose und politischer Steuerung betrachtet werden. Dabei liegt der Fokus auf der Frage, inwieweit Planungsprämissen, die aus den oben skizzierten Rahmenbedingungen abgeleitet werden, innerhalb der Verfahren im Hinblick auf den daraus resultierenden Netzausbaubedarf bzw. im Hinblick auf eine ganzheitliche Optimierung des Stromversorgungssystems auf den Prüfstand gestellt werden können. Dazu wird zunächst (3.1) das 2011 neu implementierte Instrumentarium der Netzausbaubedarfsplanung in seinen Grundzügen kurz vorgestellt. Darauf aufbauend wird die bisherige Planungspraxis diskutiert. Besonders hervorgehoben werden dabei Szenarien als ein zentrales Element der Bedarfsplanung, mit denen die zukünftigen Netznutzungen prognostiziert werden (3.2). Ein weiterer Aspekt sind planerische Abwägungsprozesse und die Berücksichtigung von Planalternativen in diesem Rahmen (3.3). Schließlich (3.4) werden Maßnahmen zur wechselseitigen Koordination von Energiepolitik und Bedarfsplanung thematisiert. Zuletzt (3.5) wird der Frage nachgegangen, wie dabei die Breite politischer Diskurse kanalisiert wird, wie diese in die Planung eingehen und welche Rolle verfahrensintegrierte Beteiligungen dabei spielen.

\subsection{Das Instrumentarium der Bedarfsplanung im Überblick}

Das Bedarfsplanungsinstrumentarium in Deutschland umfasst eine Kaskade aus drei einzelnen Verfahren:

- das Szenariorahmenverfahren gemäß § 12a EnWG,

- das Netzentwicklungsplanverfahren gemäß der $\S \S 12 b$ bis $12 \mathrm{~d}$ und 17 EnWG sowie

- das abschließende Bundesbedarfsplanverfahren gemäß $\S 12 \mathrm{e}$ EnWG.

Mit Erlass des Bundesbedarfsplangesetzes stellt der Bundesgesetzgeber auf der Grundlage der Netzentwicklungspläne onshore und offshore für die im Bundesbedarfsplan enthaltenen Vorhaben die energiewirtschaftliche Notwen- 
digkeit und den vordringlichen Bedarf verbindlich für alle nachfolgenden Planungsschritte fest.

Der Szenariorahmen umfasst mindestens drei Szenarien, die jeweils für einen mindestens zehn- bis höchstens fünfzehnjährigen Planungshorizont die Bandbreite der wahrscheinlichen Entwicklung im Rahmen der mittel- und langfristigen energiepolitischen Ziele der Bundesregierung abdecken. Ein weiteres Szenario bezieht sich auf einen mindestens fünfzehn- und höchstens zwanzigjährigen Zeitraum. Der Szenariorahmen beruht auf angemessenen Annahmen zur Erzeugung, Versorgung und zum Verbrauch von Strom sowie zum Austausch von Strom mit anderen Ländern. Die Netzentwicklungspläne bauen auf den genehmigten Szenariorahmen auf und enthalten alle wirksamen Maßnahmen zur bedarfsgerechten Optimierung, Verstärkung und zum Ausbau der Übertragungsnetze, die spätestens zum Ende des Betrachtungszeitraums für einen sicheren und zuverlässigen Netzbetrieb erforderlich sind. Zu berücksichtigen sind dabei geplante Investitionsvorhaben der europäischen Netzinfrastruktur, die unter anderem in einem gemäß Art. 8 der Verordnung (EG) Nr. 714/2009 alle zwei Jahre zu erstellenden sogenannten „Ten-Year Network Development Plan (TYNDP)“" zusammengefasst sind. Sowohl der Szenariorahmen als auch die Netzentwicklungspläne werden gemeinsam von den Übertragungsnetzbetreibern entworfen, öffentlich konsultiert und anschließend von der Bundesnetzagentur geprüft und genehmigt.

Die Übertragungsnetzbetreiber sind gemäß § 12b Abs. 4 EnWG verpflichtet darzulegen, ,aus welchen Gründen der Netzentwicklungsplan nach Abwägung mit den geprüften, in Betracht kommenden anderweitigen Planungsmöglichkeiten gewählt wurde“. Für die Netzentwicklungspläne erstellt die Bundesnetzagentur regelmäßig einen Umweltbericht, der den Anforderungen des § 14g UVPG entsprechend eine Alternativenprüfung enthalten muss, die Bestandteil jeder strategischen Umweltprüfung ist (vgl. Callies/Dross 2013: 76 f.).

Aufgrund der Unsicherheit der Zukunftsprojektionen ist gesetzlich vorgegeben, dass Szenariorahmen und Netzentwicklungspläne kontinuierlich in einem Rhythmus von zwei Jahren erstellt werden. Der Szenariorahmen ist ,jeweils an die aktuellen technischen und politischen Entwicklungen und an die gesellschaftlichen Ansprüche anzupassen“, soweit diese „von den vorangegangenen Grundannahmen abweichen“" (Heimann 2013: 62, Rn. 11). Die Bundesnetzagentur übermittelt die Netzentwicklungspläne gemäß $\S 12 \mathrm{e}$ Abs. 1 EnWG als Entwurf mindestens alle vier Jahre der Bundesregierung als Bedarfsplanentwurf. Die Bundesregierung legt den Entwurf des Bundesbedarfsplanes mindestens alle vier Jahre dem Bundesgesetzgeber vor.

Der Bedarfsplanungsprozess auf der Grundlage der EnWG-Novelle 2011 wurde erstmalig mit dem Entwurf des Szenariorahmens zum Netzentwicklungsplan (NEP)
2012 durch die Übertragungsnetzbetreiber im Juli 2011 offiziell eingeleitet (vgl. ÜNB 2011). Insgesamt liegen seitdem (Stand: März 2016) für vier Verfahrensdurchgänge genehmigte Szenariorahmen und Netzentwicklungspläne vor. Ende Juli 2013 wurde der erste Verfahrensdurchgang der Bedarfsermittlung und -feststellung mit Inkrafttreten des ersten Bundesbedarfsplangesetzes vom 23. Juli 2013 (BGB1. I : 2543) abgeschlossen.

\subsection{Szenarien der zukünftigen Netznutzung als Basis für die Ermittlung des Netzausbaubedarfs}

Die Basis für die Bedarfsermittlung bilden Szenarien, die für den Prognosezeitraum die zukünftige Netznutzung entlang verschiedener Entwicklungspfade beschreiben sollen, um auf dieser Grundlage anschließend die zukünftige Netzbelastung modellieren und Maßnahmen zur vermuteten Engpassbeseitigung planen zu können. Die Netznutzungsszenarien sind das Ergebnis mehrerer methodischer Zwischenschritte:

- die Festlegung sogenannter „Mantelzahlen“, die bundesweit aggregierte Kraftwerkskapazitäten bzw. Netzhöchstlasten bzw. Jahresstromverbräuche repräsentieren, für jedes Szenario des Szenariorahmens,

- die Festlegung von Rahmendaten für die sogenannte „Marktsimulation“ (Prognosedaten zur Entwicklung der Brennstoff- oder $\mathrm{CO}_{2}$-Zertifikatspreise bzw. zu Netznutzungen in europäischen Nachbarländern),

- die Regionalisierung der Mantelzahlen, mit der die bundesweit aggregierten Kraftwerkskapazitäten bzw. Netzhöchstlasten räumlich verteilt und letztendlich einzelnen Netzknoten zugeordnet werden, und

- die Marktsimulation, anhand derer die Verbrauchslasten bzw. der Kraftwerks- und Speichereinsatz im zeitlichen Verlauf bezogen auf ein Referenzjahr modelliert werden.

Nur die beiden ersten Zwischenschritte sind Gegenstand des Szenariorahmens. Die Regionalisierung und die Marktsimulation sind dagegen dem Netzentwicklungsplanverfahren zugeordnet. Zusammen mit der Festlegung der Mantelzahlen beeinflussen sie die Netzausbaubedarfsberechnung entscheidend (vgl. BNetzA 2011: 26 ff.; BNetzA 2012b: 16 ff.; ÜNB 2012: 18 ff.). Erst mit Fertigstellung des Netzentwicklungsplanentwurfs liegen also vollständige Netznutzungsszenarien vor. Auf diese Weise werden ungeachtet der Kritik verschiedener Konsultationsteilnehmer (vgl. BNE 2011: 1; BUND 2011: 1; MVV Energie AG 2011: 1; RWE Verteilnetz GmbH 2011: 1) zwei der für die Szenariengestaltung entscheidenden Weichenstellungen zu einem Element der technischen Maßnahmenplanung. Dies bedeutet auch, dass die Eingangsparameter für die Regionalisierung und die Marktsimulation nicht den gesetzlichen Anforderungen gemäß § 12a EnWG unterliegen, 
wonach die Szenarien die „Bandbreite wahrscheinlicher Entwicklungen im Rahmen der mittel- und langfristigen energiepolitischen Ziele der Bundesregierung" abdecken sollen ( $\$ 12 \mathrm{a}$ Abs. 1 S. 2 EnWG). Dementsprechend entfällt die Verpflichtung, für die Prognose der Standortverteilungen oder des Kraftwerks- und Speicherbetriebs Parametervariationen in die Planung einzubringen.

Eine Rückkopplung zwischen dem Szenariorahmen- und dem Netzentwicklungsplanverfahren innerhalb eines Verfahrensdurchgangs ist vom Gesetzgeber nicht vorgesehen. Eine Nachjustierung der Netznutzungsszenarien auf Basis des resultierenden Netzausbaubedarfs und auf der Grundlage angepasster Planungsprämissen ist damit allenfalls in einem späteren Verfahrensdurchgang möglich.

\subsection{Bedarfsfeststellung als Abwägungsentscheidung}

Für den ersten Netzentwicklungsplan, der auch die Grundlage für das erste Bundesbedarfsplangesetz 2013 war, hatten die Übertragungsnetzbetreiber die Maßnahmen auf der Basis eines sogenannten „Leitszenarios“ geplant, das aus den drei Szenarien des Szenariorahmens ausgewählt worden war. Dieser Netzentwicklungsplan wurde von der Bundesnetzagentur bestätigt, ohne dass zuvor im Rahmen der strategischen Umweltprüfung (SUP) eine Alternativenprüfung stattgefunden hatte (vgl. BNetzA 2012c: 52 ff.).

Der Verzicht auf die Alternativenprüfung ist auf deutliche Kritik gestoßen. Hermes (2014: 266) sah die gesetzgebenden Organe angesichts dessen ,in einer Ratifikationslage ohne Entscheidungsalternative“. Gemäß Callies/Dross (2013: 78) wäre die Bundesnetzagentur - auch europarechtlich - schon im ersten Verfahrensdurchgang verpflichtet gewesen, eine Alternativenprüfung durchzuführen und dabei auch konzeptionelle Alternativen in den Blick zu nehmen. Dabei wäre es - nach Auffassung von Callies/Dross (2013: 79) - auch zulässig gewesen, Alternativbetrachtungen zu der Frage anzustellen, ,an welchen Orten Strom in welcher Menge aus welchen Quellen voraussichtlich in das Netz eingespeist werden [sic!]“. Entsprechende Überlegungen seien beim Szenariorahmen erkennbar nicht vorgenommen worden. Dass sie gänzlich unterblieben, sei jedoch nicht im Sinne der SUP-Richtlinie (Richtlinie über die strategische Umweltverträglichkeitsprüfung). $\mathrm{Zu}$ empfehlen sei, im Rahmen der Bedarfsplanung mindestens ein Szenario zu entwickeln, ,das in seinen Annahmen stärker von den vorliegenden Szenarien abweicht", um dieses dann zum Gegenstand der Alternativenprüfung zu machen (Callies/Dross 2013: 79).

\subsection{Ansätze zur flexiblen wechselseitigen Anpassung zwischen Bedarfsplanung und energiepolitischer Rahmensetzung}

Die Planungsträger haben während des ersten Verfahrensdurchgangs immer wieder betont, dass grundsätzlich alle Netznutzungsentscheidungen dem Wettbewerb unterliegen und sich dem netzplanerischen Zugriff entziehen (vgl. BNetzA 2011: 26, 345; ÜNB 2012: 178, 181). Sie haben dementsprechend Forderungen von Konsultationsteilnehmern zurückgewiesen, potenzielle staatliche Steuerungseingriffe als eine Option zur Vermeidung von Netzengpässen bzw. zur ganzheitlichen Optimierung des Stromversorgungssystems in die Bedarfsplanung zu integrieren (vgl. BEE 2011: 3; BfN 2011: 3; BUND 2011: 1; DUH 2011: 3; NABU 2011: 2; ÖI 2011: 4; UBA 2011: 2; BMELV 2012: 7 f.; Krawinkel 2012: 461; Landkreis Lüneburg 2012: 2). Als Begründung dafür wurde regelmäßig angeführt, dass die gesetzten Input-Parameter strikt an politisch-rechtlichen Status-quo-Bedingungen auszurichten seien (vgl. BNetzA 2011: 36; BNetzA 2012b: 363; ÜNB 2012: 181).

In den nachfolgenden Verfahrensdurchgängen geriet die Netzplanung jedoch aufgrund kurzfristiger Änderungen politischer Rahmenbedingungen zunehmend unter Anpassungsdruck. Wichtige Zäsuren waren unter anderem der Regierungswechsel auf Bundesebene 2013 und die daraus resultierende Neuausrichtung der Energiepolitik, die durch zahlreiche einschlägige Gesetzgebungsvorhaben flankiert werden sollte (vgl. Große Koalition 2013; BMWi 2015). Aufgrund sehr unterschiedlicher zeitlicher Taktungen war es nicht möglich, die Bedarfsplanung mit laufenden politischen Willensbildungs- bzw. Gesetzgebungsprozessen vollständig zu synchronisieren. Dies erforderte es, die Prozesse der Abstimmung zwischen energiepolitischen Eingriffen und Netzplanung flexibler zu gestalten.

Im Zuge der Genehmigung der Szenariorahmen für die Zieljahre 2023 und 2024 hatte die Bundesnetzagentur den Übertragungsnetzbetreibern zusätzlich sogenannte ,Sensitivitätsanalysen“ aufgegeben, die parallel zur Netzentwicklungsplanung durchgeführt werden sollten. Dazu hat sie für jede Sensitivitätsanalyse in Bezug auf einzelne Parameter des sogenannten Leitszenarios jeweils eine bestimmte Parametervariation vorgegeben. Die Wirkungen der so veränderten Planungsprämissen auf den Netzausbaubedarf sollten die Übertragungsnetzbetreiber mithilfe gesonderter Netzberechnungen überprüfen, ohne damit zunächst die laufende Netzplanung unmittelbar zu beeinflussen. Die Parametervariationen wurden gezielt zur Analyse der Wirkungen von beabsichtigten energiepolitischen Strategieänderungen im Vorgriff auf deren gesetzgeberische Implementierung eingesetzt, um sie dann gegebenenfalls in nachfolgenden 
Verfahrensdurchgängen auch formell in der Planung zu berücksichtigen (vgl. BNetzA 2014a: 57).

Die Einführung der Spitzenkappung für Einspeiseleistungen aus Fotovoltaik- und Onshore-Windkraftanlagen war Gegenstand einer Sensitivitätsanalyse zu den Netzentwicklungsplänen für die Zieljahre 2023 und 2024. Nachdem die Analysen ergeben hatten, dass eine solche Maßnahme zu einer deutlichen Reduktion des Netzausbaubedarfs beitragen könnte, wurde diese dem Szenariorahmen für den Netzentwicklungsplan 2015 zugrunde gelegt. Erstmals kam damit eine Planungsprämisse zum Tragen, die explizit darauf ausgerichtet war, den Netzausbaubedarf zu reduzieren und die zudem durch den zu diesem Zeitpunkt gültigen Rechtsrahmen noch nicht abgedeckt war (vgl. BNetzA 2014a: III). Erst im Januar 2016 hat die Bundesregierung auf der Grundlage der positiven Wirkungsprognose für diese Maßnahme einen Referentenentwurf für das sogenannte „Strommarktgesetz“ vorgelegt, der die Implementierung der „Spitzenkappung“ vorsieht (vgl. Deutscher Bundestag 2016).

\subsection{Politische Steuerung der Bedarfsplanungsprozesse}

Umweltverbände, die sich an Konsultationen der Planentwürfe beteiligt hatten, haben in ihren Stellungnahmen die Einführung der Sensitivitätsanalysen sehr begrüßt. Allerdings kritisierten sie die wenig transparenten Entscheidungswege und -gründe für Auswahl und Konzeption der sogenannten „Sensitivitäten“. Sie forderten, Sensitivitätsbetrachtungen ,in einem breiten gesellschaftlichen Diskussionsprozess“ (Germanwatch 2013a: 2) und ,im Dialog mit Experten und Vertretern der Zivilgesellschaft" zu konzipieren und auszugestalten (DUH 2013: 8). Dies solle dazu beitragen, im Sinne eines ,lernenden Systems“ die politische Meinungsbildung zu befördern (vgl. Germanwatch 2013b: 2).

Zur Diskussion der inhaltlichen Ausrichtung der Sensitivitätsanalysen hat die Bundesnetzagentur im Zuge der Konsultation des Szenariorahmenentwurfs für das Zieljahr 2025 die Teilnehmer befragt und dabei auch um Hinweise gebeten, „falls weitere Sensitivitätsberechnungen für den Netzentwicklungsplan 2025 für sinnvoll erachtet werden“ (BNetzA 2014c: 9). Den Darstellungen der Bundesnetzagentur entsprechend sind zahlreiche Konsultationsteilnehmer dieser Aufforderung nachgekommen. In vielen Stellungnahmen wurde der Wunsch geäußert, Maßnahmen mithilfe von Sensitivitätsanalysen zu untersuchen, die darauf ausgerichtet sein sollten, den Netzausbaubedarf zu minimieren. Einzelne Vorschläge zielten darauf ab zu analysieren, mit welchen Maßnahmen oder Rahmenbedingungen bei Einhaltung der energiepolitischen Ziele sich der Netzausbaubedarf auf ein bestimmtes Niveau begrenzen lasse (vgl. BNetzA 2014a: 15, 38).
Viele andere Vorschläge bezogen sich auf die bedarfsreduzierende Wirkung einer dezentralen Energiewende mit verbrauchsnaher Erzeugung (vgl. BNetzA 2014b: 48) oder einer netzdienlichen Standortwahl für Kraftwerke (vgl. BNetzA 2014a: 4, 14), einer verstärkten Speicherung (BNetzA 2014a: 47) oder eines integrierten Strom-, Wärme- und Mobilitätsmarktes (vgl. BNetzA 2014a: 13). Zudem solle ein technologischer Durchbruch der Fotovoltaik weit jenseits der bisher in den Szenarien prognostizierten Kapazitäten ,gerade mit Blick auf die längerfristige Perspektive (2035)“ als Sensitivität in Deutschland untersucht werden. Dabei seien auch die Effekte eines „PV-BatteryBreakthrough"-Szenarios auf das Stromnetz zu betrachten (vgl. BNetzA 2014a: 13 f.).

Die Bundesnetzagentur hat bislang keinen der genannten Vorschläge aufgegriffen. Als Voraussetzung für die Untersuchung potenzieller Steuerungseingriffe im Rahmen von Sensitivitätsanalysen sollte vielmehr gelten, dass entsprechende Gesetzgebungsvorhaben ,sich hinreichend konkret abzeichnen“ und dass ,der Konsens in Politik, Fachwelt und Gesellschaft so groß ist, dass mit einer baldigen rechtlichen Verankerung gerechnet werden muss" (vgl. BNetzA 2014a: 56 f.).

Dieses Beispiel zeigt, wie die Bundesnetzagentur die Planungsverfahren dominiert und dabei auch Entscheidungen trifft, die einen deutlich politischen Charakter aufweisen. Hermes (2014) stellt die ,demokratische Legitimation" solcher Entscheidungen in der Letztverantwortung einer Behörde grundsätzlich in Frage und fordert stattdessen einen ,transparenten politischen Prozess in der Hand der politischen Organe“ (Hermes 2014: 265 f.).

Vermutlich wird die Bundesnetzagentur bei ihren Entscheidungen durch die „Plattform Energienetze“ unterstützt, die der Bundeswirtschaftsminister schon 2010 als „Plattform zukunftsfähige Energienetze“ gegründet hatte. Dieses Gremium, das mit den ,wesentlichen Interessenvertreter(n) - Netzbetreiber, Bundes- und Länderinstitutionen sowie Wirtschafts-, Verbraucher- und Umweltverbände“ besetzt ist, hatte sowohl an der Konzeption des Planungsinstrumentariums im Zuge der EnWG-Novelle 2011 als auch an der des ersten Szenariorahmens maßgeblich mitgewirkt (ÜNB 2011: 3; vgl. dena 2012).

\section{Fazit}

Die hier vorgelegten Analysen zeigen, dass Liberalisierung und Binnenmarktintegration einen großräumigen Ausgleich zwischen der Erzeugung und dem Verbrauch von Energie befördern. Das Strommarktdesign bietet bislang weder Anreize für eine an Verbrauchsstandorten orientierte Kraftwerksansiedlung noch für einen Anlagenbetrieb, der auf eine regionale Bedarfsdeckung ausgerichtet ist. Die zuletzt 
durch die Bundesregierung im Verein mit europäischen Nachbarländern eingeleitete Verlagerung der Gewährleistungsverantwortung für die Versorgungssicherheit vom Nationalstaat auf die transnationale Ebene führt außerdem zu einer Neuordnung des europäischen Kraftwerksparks, die zusammen mit der Harmonisierung der Ausbauförderung der Erneuerbaren Energien (EE) die Konzentration der Stromerzeugung auf Gunststandorte nach rein betriebswirtschaftlichen Kriterien begünstigt.

Unter den gegebenen Markt- und Netznutzungsregularien bedingt jeglicher nennenswerte Zubau von fluktuierenden erneuerbaren Energien-Kapazitäten (fEE) einen Ausbau der Übertragungsnetze. Eine Konzentration dieses Zubaus auf verbrauchsnahe Standorte würde für sich genommen voraussichtlich allenfalls zu einer geringen Reduktion, jedoch in Teilen zu einer räumlichen Verschiebung des Netzausbaubedarfs hin zu Verbrauchszentren führen. Nur durch den zusätzlichen lastnahen Ausbau der Back-upStromerzeugung z. B. durch erdgasbetriebene Kraft-Wärme-Kopplungsanlagen, vor allem aber durch einen erhöhten Grad von Leistungsautarkie, bei der Verbrauch und Erzeugung möglichst schon vor Ort ausbalanciert werden, wäre eine signifikante Verminderung des Übertragungsnetzausbaubedarfs zu erwarten.

Konzepte des regionalen Ausgleichs verursachen einen höheren Aufwand für die Stromspeicherung und für die Bereitstellung von Erzeugungskapazitäten. Der regionale Ausgleich bietet andererseits den Vorteil, Maßnahmen zur Integration der Stromversorgung, der Wärmeversorgung und der Versorgung des Verkehrssektors vor Ort leichter und gegebenenfalls auch kostengünstiger umsetzen zu können. Ein zukünftig höherer Erdkabelanteil im Übertragungsnetz dürfte zudem den Netzausbau spürbar verteuern und verringert dadurch den Kostenvorteil zentraler Transformationspfade.

Allerdings erfordert eine Implementation des zellularen Ansatzes zunächst die technische Erprobung und die Etablierung von geeigneten Geschäftsmodellen. Er wird schon aus diesem Grund bei der Netzausbauplanung aktuell nicht berücksichtigt (vgl. BNetzA 2011: 77 ff.). Vor dem Hintergrund der Probleme mit der Netzführung angesichts der zunehmenden Komplexität aufgrund von hohen Anteilen der Einspeisung auf unteren Netzebenen könnte er aber mittelfristig an Bedeutung gewinnen.

Bislang werden die Übertragungsnetze geplant, ohne dabei die Verteilnetzebene voll umfänglich mit einzubeziehen. Dezentral orientierte Transformationsansätze bedingen aber insbesondere einen Umbau der Verteilnetze. Insofern wäre es sinnvoll, in der Bedarfsplanung diesen Umbau auf Verteilnetzebene stärker zu berücksichtigen.

Ein weitgehend engpassfreier Netzausbau, wie ihn die derzeitige Marktordnung und Netzregulierung erfordert, führt zu Pfadabhängigkeiten, indem er dezentralen Ansät- zen der Energiesystemtransformation den wirtschaftlichen Boden entzieht. Insoweit stellt sich die Frage der mittelund langfristigen Zielorientierung für die Energiesystemtransformation. Allerdings existiert in Deutschland weder eine Bundesraumordnung noch eine Energiefachplanung, wo explizit die räumliche Ausgestaltung des Energiesystems verhandelt werden könnte. Die entscheidenden Rahmenbedingungen werden stattdessen implizit über die Marktordnung und die Netzregulierung gesetzt.

Die Netzplanung integriert diese energiepolitischen Rahmensetzungen als Planungsprämissen insbesondere für die Regionalisierung und die Marktsimulation, die beide den Netzausbaubedarf maßgeblich beeinflussen. Diese beiden Planungsschritte sind bislang nicht Gegenstand des Szenariorahmenverfahrens, das in dieser Hinsicht stärker geöffnet werden sollte. Die Standortverteilung der Stromerzeugung, der Speicherung und des Verbrauchs sollte ebenso wie die Markt- und Netzregularien, die den Betrieb dieser Anlagen steuern, integraler Gegenstand des Szenariorahmenverfahrens sein. Dadurch würden diese Parameter bereits in den Entwürfen enthalten sein und würden gleichzeitig den Konsultationen sowie der Prüfung und Genehmigung durch die Bundesnetzagentur zugänglich gemacht werden. Dies würde die Transparenz der Bedarfsplanung erhöhen und es ermöglichen, vielfältigere Optionen für die Parametervariationen zur Modellierung verschiedener konsistenter Entwicklungspfade zu berücksichtigen.

Sinnvoll wäre es außerdem, regelmäßig Szenarien in die Alternativenprüfung einzubeziehen, die auf eine Reduzierung des Netzausbaubedarfs ausgerichtet sind. Damit ergäbe sich eine Basis für eine vergleichende Bewertung verschiedener Entwicklungsoptionen unter Zugrundelegung der Ziele gemäß § 1 EnWG. Dazu gehören die Versorgungssicherheit (einschließlich dem Schutz der Infrastruktur bei Vulnerabilität), die volkswirtschaftliche Effizienz sowie die Umwelt- und Sozialverträglichkeit der Stromversorgung.

Die Bundesnetzagentur hat die Sensitivitätsanalyse als ein Instrument etabliert, das dazu dient, politische Handlungsoptionen im Hinblick auf ihre Wirkungen auf den Netzausbaubedarf im laufenden Bedarfsplanverfahren zu überprüfen. Die kürzlich gesetzlich implementierte Spitzenkappung für die Wind- und Solarstromerzeugung, die durch eine Sensitivitätsanalyse vorbereitet worden ist, ist ein erster Schritt hin zur Öffnung der Netzplanung, die es erlaubt, auch Maßnahmen bewusst einzubeziehen, die zur Verminderung des Netzausbaubedarfs beitragen können. Dieses Instrument könnte auch genutzt werden, um auf der Basis fundierter Wirkungsanalysen unterschiedliche Transformationspfade systematisch in die netzplanerische und zugleich energiepolitische Abwägung einzustellen und so einen ganzheitlichen Steuerungsansatz mit integrierter Öffentlichkeitsbeteiligung zu verfolgen. 
Allerdings kanalisiert die Bundesnetzagentur Konzepte zur Ausgestaltung dieser Überprüfung, indem sie nur Vorschläge aufnimmt, für die sie einen breiten Konsens in Politik, Fachwelt und Gesellschaft feststellt. Diese Einschränkung lässt die Tatsache unberücksichtigt, dass ein solcher Konsens vor allem dann erzielt werden kann, wenn der Erfolg entsprechender Maßnahmen verifiziert wurde und quantifizierbar ist. Zudem werden für die Sensitivitätsanalysen jeweils nur einzelne Parametervariationen zugelassen, obwohl gerade Maßnahmenkombinationen einen hohen Einfluss auf den Netzausbaubedarf erwarten lassen.

Die Übertragungsnetzbetreiber sollen beim Entwurf der Szenariorahmen dem gesetzlichen Auftrag folgend den energiepolitischen Zielrahmen abstecken, innerhalb dessen sie die Bandbreite wahrscheinlicher Entwicklungen der Stromerzeugung, des Stromverbrauchs und der Stromversorgung prognostizieren. Sie sind aber ebenso wie die Bundesnetzagentur kaum dafür prädestiniert, dem politischen Willen der Bundesregierung Ausdruck zu verleihen. Daher kommt der „Plattform zukünftige Energienetze“, in der sowohl Bundes- und Landesregierungen vertreten sind, die Funktion zu, die Planungsprozesse politisch von außen zu steuern.

Da die Öffentlichkeit keinen Einblick in deren interne Diskussions- und Entscheidungsprozesse hat, besteht hier ein erhebliches Transparenzdefizit. Es ist deshalb zu empfehlen, die Arbeit der Plattform der Öffentlichkeit besser zugänglich zu machen. Dazu sollten gegebenenfalls Sitzungsprotokolle oder Tätigkeitsberichte publiziert werden.

\section{Literatur}

Adam, K.; Agsten, M.; Benedix, C.; Berg, V.; Blug, C.; Brochtrop, M. (2013): Aktive Energienetze im Kontext der Energiewende. Anforderungen an künftige Übertragungs- und Verteilungsnetze unter Berücksichtigung von Marktmechanismen. Frankfurt/Main.

ARL - Akademie für Raumforschung und Landesplanung (Hrsg.) (2014): Leitbilder und Handlungsstrategien der Raumentwicklung in Deutschland 2013. Hannover. = Positionspapier aus der ARL, 96.

Bauknecht, D.; Vogel, M.; Funcke, S. (2015): Energiewende - Zentral oder dezentral? Diskussionspapier im Rahmen der Wissenschaftlichen Koordination des BMBF Förderprogramms: „Umwelt- und Gesellschaftsverträgliche Transformation des Energiesystems“. Freiburg im Breisgau.

BdEW - Bundesverband der Energie- und Wasserwirtschaft e.V. (Hrsg.) (2012): Stellungnahme zum Bericht der Plattform Erneuerbare Energien an die Bundeskanzlerin und die Ministerpräsidentinnen und Ministerpräsidenten der Länder vom 28.09.2012. Berlin.

BEE - Bundesverband Erneuerbare Energien e. V. (2011): Stellungnahme zum Konsultationsverfahren der BNetzA über die Eingangsdaten des Szenariorahmens für den Netzentwicklungsplan 2012. Berlin.

Benz, T.; Dickert, J.; Erbert, M.; Erdmann, N.; Johae, C.; Katzenbach, B. (2015): Der zellulare Ansatz. Grundlage einer erfolgreichen, regionenübergreifenden Energiewende. Studie der Energietechnischen Gesellschaft im VDE (ETG). Frankfurt/Main.
BfN - Bundesamt für Naturschutz (2011): Konsultation des Szenariorahmens zur Erstellung des ersten 10-Jahres-Netzentwicklungsplanes der Übertragungsnetzbetreiber nach § 12a EnWG. Bonn.

Birkner, P. (2014): Wege zu einer gelungenen Energiewende. Prinzipien für eine effiziente, effektive und erfolgreiche Umsetzung. In: VDE Informationen 03/2014, 1-3.

BMELV - Bundesministerium für Ernährung, Landwirtschaft und Verbraucherschutz (2012): Stellungnahme des BMELV zum Netzentwicklungsplan (NEP) und der vorläufigen Umweltprüfung. Berlin.

BMVI - Bundesministerium für Verkehr und digitale Infrastruktur (2015): Regionale Energiekonzepte als strategisches Instrument der Landes- und Regionalplanung. Berlin. = BMVI-Online-Publikation 09/2015.

BMWi - Bundesministerium für Wirtschaft und Energie (2014): Ein Strommarkt für die Energiewende. Diskussionspapier des Bundesministeriums für Wirtschaft und Energie (Grünbuch). Berlin.

BMWi - Bundesministerium für Wirtschaft und Energie (2015): Zentrale Vorhaben Energiewende für die 18. Legislaturperiode (Fortschreibung der 10-Punkte-Energie-Agenda des BMWi). Berlin.

BNE - Bundesverband Neue Energieanbieter (Hrsg.) (2011): BNEStellungnahme zum Szenariorahmen für den Netzentwicklungsplan 2012. Eingangsdaten der Konsultation. Berlin.

BNetzA - Bundesnetzagentur (2011): Genehmigungsschreiben Szenariorahmen zum Netzentwicklungsplan 2012 vom 20. Dezember 2011. Bonn.

BNetzA - Bundesnetzagentur (2012a): Genehmigungsschreiben Szenariorahmen zum Netzentwicklungsplan 2013 vom 30. November 2012. Bonn.

BNetzA - Bundesnetzagentur (2012b): Bestätigung Netzentwicklungsplan Strom 2012 durch die Bundesnetzagentur für Elektrizität, Gas, Telekommunikation, Post und Eisenbahnen vom 25. November 2012. Bonn.

BNetzA - Bundesnetzagentur (2012c): Umweltbericht zum Bundesbedarfsplan-Entwurf. Bonn.

BNetzA - Bundesnetzagentur (2013): Genehmigungsschreiben Szenariorahmen zum Netzentwicklungsplan 2014 vom 30. August 2013. Bonn.

BNetzA - Bundesnetzagentur (2014a): Genehmigungsschreiben Szenariorahmen zum Netzentwicklungsplan 2015 vom 19. Dezember 2014. Bonn.

BNetzA - Bundesnetzagentur (2014b): Schwerpunkt der Diskussionen. Workshop zum Szenariorahmen 2025 am 28. Mai 2014 in Berlin. Bonn.

BNetzA - Bundesnetzagentur (2014c): Begleitdokument zur Konsultation des Szenariorahmens 2025. Stand: 12.05.2014. Bonn.

Bucksteeg, M.; Niesen, L.; Himmes, P.; Schober, D.; Weber, C.; Baumgart, B.; Plöger, T.; Willemsen, D.; Nailis, D.; Schuffelen, L.; Bittner, M. (2014): Marktdesign für zukunftsfähige Elektrizitätsmärkte unter besonderer Berücksichtigung der vermehrten Einspeisung von erneuerbaren Energien - DESIRE - Endbericht. Studie der Universität Duisburg-Essen, Lehrstuhl für Energiewirtschaft (EWL), der Trianel GmbH und des BET Büro für Energiewirtschaft und technische Planung GmbH. Duisburg, Aachen.

BUND - Bund für Umwelt und Naturschutz Deutschland e. V. (Hrsg.) (2011): Stellungnahme zum Szenariorahmen für den Netzentwicklungsplan 2012. Berlin.

Bundesverband der Bürgerinitiativen gegen den SüdLink (2014): Alles auf Null .... http://buergerinitiativen-gegen-suedlink.de/?page_ id $=27(11.10 .2014)$.

Calliess, C.; Dross, M. (2013): Alternativenprüfungen im Kontext des Netzausbaus - Überlegungen mit Blick auf die strategische Umweltprüfung des Bundesbedarfsplans Übertragungsnetze. In: Zeitschrift für Umweltrecht 24, 2, 76-82.

CONSENTEC - Consulting für Energiewirtschaft und -technik GmbH; Frontier economics Ltd. (2008): Notwendigkeit und Ausgestal- 
tung geeigneter Anreize für eine verbrauchsnahe und bedarfsgerechte Errichtung neuer Kraftwerke. Ein Gutachten für das Bundesministerium für Wirtschaft und Technologie. Aachen, London.

dena - Deutsche Energie-Agentur GmbH (2012): AG Netzentwicklungsplan. Szenariorahmen für den Netzentwicklungsplan 2012. www.dena.de/projekte/energiesysteme/ag-netzentwicklungsplan. html (11.03.2015).

Deutscher Bundestag (2011): Gesetzentwurf der Fraktionen der CDU/CSU und FDP. Entwurf eines Gesetzes zur Neuregelung energiewirtschaftsrechtlicher Vorschriften. Bundestagsdrucksache 17/6072 vom 06.06.2011. Berlin.

Deutscher Bundestag (2016): Gesetzentwurf der Bundesregierung. Entwurf eines Gesetzes zur Weiterentwicklung des Strommarktes (Strommarktgesetz). Bundestagsdrucksache 18/7317 vom 20.01.2016. Berlin.

DUH - Deutsche Umwelthilfe (2011): Stellungnahme zum Entwurf des Szenariorahmens zum Netzentwicklungsplan 2012. Berlin.

DUH - Deutsche Umwelthilfe (2013): Stellungnahme zum Szenariorahmen für die Netzentwicklungspläne Strom 2014. Erster Entwurf der Übertragungsnetzbetreiber vom 28. März 2013. Bonn.

ENTSO-E - European Network of Transmission System Operators for Electricity (2010): Ten Years Network Development Plan (TYNDP) 2010. Brüssel.

EU-Kommission (2014): Leitlinien für staatliche Umweltschutz- und Energiebeihilfen 2014-2020 (2014/C 200/01). In: Amtsblatt der Europäischen Union 57, C 200, 1-55.

Fuchs, B.; Mittelstaedt, M.; Natemeyer, H.; Özalay, B.; Surmann, Y.; Hackmann, M.; Hörpel, B. (2012): Szenarien für eine langfristige Netzentwicklung. Schlussbericht. Studie im Auftrag des Bundesministeriums für Wirtschaft und Technologie der P3 energy and storage GmbH und des Instituts für Hochspannungstechnik (IFHT) der RWTH Aachen. Aachen.

Fürstenwerth, D.; Bock, N.; Tersteegen, B.; Pape, C. (2013): Kostenoptimaler Ausbau der Erneuerbaren Energien in Deutschland. Ein Vergleich möglicher Strategien für den Ausbau von Wind- und Solarenergie in Deutschland bis 2033. Studie herausgegeben von Agora Energiewende, Fraunhofer Institut für Windenergie und Energiesystemtechnik (IWES) und CONSENTEC - Consulting für Energiewirtschaft und -technik GmbH. Berlin.

Germanwatch (2013a): Stellungnahme zum Netzentwicklungsplan Strom 2013 - Entwurf der Übertragungsnetzbetreiber. Berlin.

Germanwatch (2013b): Stellungnahme zum Szenariorahmen für den Netzentwicklungsplan Strom 2014. Berlin.

Groscurth, H.-M.; Bode, S. (2012): Zielkonflikte in der Stromerzeugung im Kontext der Energiewende. Studie des Arrhenius Institut für Energie- und Klimapolitik. Hamburg. = Discussion Paper, 7.

Große Koalition (2013): Deutschlands Zukunft gestalten. Koalitionsvertrag zwischen CDU, CSU und SPD. 18. Legislaturperiode. Berlin.

Grünwald, R. (2015): Moderne Stromnetze als Schlüsselelement einer nachhaltigen Energieversorgung. Berlin. = Arbeitsbericht des Büro für Technikfolgen-Abschätzung beim Deutschen Bundestag (TAB), 162.

Haucap, J.; Pagel, B. (2014): Ausbau der Stromnetze im Rahmen der Energiewende: Effizienter Netzausbau und effiziente Struktur der Netznutzungsentgelte. Düsseldorf. = DICE Ordnungspolitische Perspektiven, 55

Heimann, U. (2013): Kommentar zu § 12a EnWG. In: Steinbach, A. (Hrsg.): NABEG/EnLAG/EnWG. Kommentar zum Recht des Energieleitungsbaus. Berlin, Boston, 59-72.

Hermes, G. (2014): Planungsrechtliche Sicherung einer Energiebedarfsplanung - ein Reformvorschlag. In: Zeitschrift für Umweltrecht 25, 5, 259-269.

Hobohm, J.; Koepp, M.; Michelsen, C.; Peter, F. (2006): Variantenvergleich Küste versus Binnenland. Ein volkswirtschaftlicher Vergleich der Kosten, Versorgungssicherheit und Umweltverträglich- keit von Kraftwerksstandorten. Endbericht der Prognos AG. Berlin.

König, C. (2013): Engpassmanagement in der deutschen und europäischen Elektrizitätsversorgung. Baden-Baden. $=$ Kartell- und Regulierungsrecht, 4.

Krawinkel, H. (2012): Der Infrastrukturausbau im Rahmen der Energiewende benötigt umfassende Planungsinstrumente. In: Zeitschrift für Neues Energierecht 16, 5, 461-465.

Landkreis Lüneburg (2012): Entwurf Netzentwicklungsplan. Konsultation. Lüneburg.

Litz, P.; Rosenkranz, G. (2015): Stromexport und Klimaschutz in der Energiewende. Analyse der Wechselwirkungen von Stromhandel und Emissionsentwicklung im fortgeschrittenen europäischen Strommarkt. Berlin.

Mautz, R.; Byzio, A.; Rosenbaum, W. (2008): Auf dem Weg zur Energiewende. Die Entwicklung der Stromproduktion aus erneuerbaren Energien in Deutschland. Eine Studie aus dem Soziologischen Forschungsinstitut Göttingen (SOFI). Göttingen.

Monopolkommission (2013): Wettbewerb in Zeiten der Energiewende. Sondergutachten der Monopolkommission gemäß $§ 62$ Abs. 1 EnWG. Bonn.

Müller, T. (2014): Der neue Beihilferahmen für erneuerbare Energien eine energiepolitische Revolution aus Brüssel? Vortrag anlässlich der 9. Internationalen Energiewirtschaftstagung „Energiesysteme im Wandel: Evolution oder Revolution?" des Instituts für Energiesysteme und Elektrische Antriebe der TU Wien (IEWT) in Wien am 13.02.2014. http://www.eeg.tuwien.ac.at/eeg.tuwien.ac.at_ pages/events/iewt/iewt2015/uploads/abstracts/A_248_Mueller_ Thorsten_12-Jan-2015_14-34.pdf (02.05.2016).

MVV Energie AG (2011): Stellungnahme in der Konsultation zum Szenariorahmen zur Erstellung von Netzentwicklungsplänen nach $\S 12 \mathrm{a}$ Abs. 2 EnWG. Mannheim.

NABU - Naturschutzbund Deutschland e.V. (2011): Stellungnahme zum Szenariorahmen für den Netzentwicklungsplan 2012. Berlin.

N-ERGIE AG (2015): Versorgungssicherheit oder EU-Binnenmarkt? Pressemitteilung vom 30.01.2015. Nürnberg.

Nicolosi, M. (2012): Notwendigkeit von Kapazitätsmechanismen. Endbericht der ECOFYS Germany GmbH. Berlin.

ÖI - Öko-Institut (2011): Zum Szenariorahmen für den Netzentwicklungsplan 2012 Elektrizitäts-Übertragungsnetze. Stellungnahme für die Konsultation der Bundesnetzagentur. Berlin.

Peter, S. (2013): Modellierung einer vollständig auf erneuerbaren Energien basierenden Stromerzeugung im Jahr 2050 in autarken, dezentralen Strukturen. Dessau-Roßlau. = Climate Change, $14 / 2013$

Plattform EE - Erneuerbare Energien (2012): Bericht der AG 3 Interaktion an den Steuerungskreis der Plattform Erneuerbare Energien, die Bundeskanzlerin und die Ministerpräsidentinnen und Ministerpräsidenten der Länder. Stand: 15.10.2012. Berlin.

r2b energy consulting GmbH; ISI - Fraunhofer Institut für System und Innovationsforschung; CONNECT Energy Economics; CONSENTEC - Consulting für Energiewirtschaft und -technik GmbH (2014): Leitstudie Strommarkt. Arbeitspaket Funktionsfähigkeit EOM und Impact-Analyse Kapazitätsmechanismen. Endbericht im Auftrag des Bundesministeriums für Wirtschaft und Energie. Köln.

RWE Verteilnetz GmbH (2011): Stellungnahme zur Konsultation eines Szenariorahmens zur Erstellung von Netzentwicklungsplänen nach $\S 12 \mathrm{a}$ Abs. 2 des neuen EnWG. Wesel.

Schuster, H. (2013): Gesamtwirtschaftliche Bewertung von Kraftwerksstandorten zur Parametrierung exogener Allokationssignale. Aachen. $=$ Aachener Beiträge zur Energieversorgung, 154 .

UBA - Umweltbundesamt (2011): Stellungnahme des Umweltbundesamtes zur Konsultation des Szenariorahmens zum Netzentwicklungsplan 2012. Dessau.

ÜNB - Übertragungsnetzbetreiber (50 Hertz Transmission GmbH; Amprion $\mathrm{GmbH}$; TenneT TSO GmbH; Transnet BW GmbH) (2011): Szenariorahmen für den Netzentwicklungsplan 2012 - 
Eingangsdaten der Konsultation. Stand: 18. Juli 2011. Bayreuth, Berlin, Dortmund, Stuttgart.

ÜNB - Übertragungsnetzbetreiber (50 Hertz Transmission GmbH; Amprion GmbH; TenneT TSO GmbH; Transnet BW GmbH) (2012): Netzentwicklungsplan Strom 2012. 2. überarbeiteter Entwurf der Übertragungsnetzbetreiber vom 15. August 2012. Bayreuth, Berlin, Dortmund, Stuttgart.

VDE - Verband der Elektrotechnik, Elektronik, Informationstechnik e. V. (2015): Energiewende von ,unten“. VDE-Studie zeigt wie Stromnetzausbau reduziert werden kann. Pressemitteilung vom 16. Juni 2015. Frankfurt/Main.
VDE - Verband der Elektrotechnik, Elektronik, Informationstechnik e. V.; ITG - Informationstechnische Gesellschaft (2010): Energieinformationsnetze und -systeme. Bestandsaufnahme und Entwicklungstendenzen. Frankfurt/Main.

Wachsmuth, J.; Petschow, U.; Brand, U.; Fettke, U.; Pissarskoi, E.; Fuchs, G.; Dickel, S.; Kljajic, M. (2015): Richtungsgebende Einflussfaktoren im Spannungsfeld von zentralen vs. dezentralen Orientierungen bei der Energiewende und Ansatzpunkte für ein Leitkonzept Resilienz. Bremen, Berlin. = Resystra Diskussionspapier, 1. 